Chronic Obstructive Pulmonary Diseases:

Journal of the COPD Foundation

\author{
Original Research
}

\title{
Expanding Post-Discharge Readmission Metrics in Patients with Chronic Obstructive Pulmonary Disease
}

Laura C. Myers, MD, MPH ${ }^{1,2}$ Carlos Camargo, DrPH, MD ${ }^{3}$ Gabriel Escobar, MD ${ }^{1,2}$ Vincent X. Liu, MD ${ }^{1,2}$

\section{Abstract}

Background: Chronic obstructive pulmonary disease (COPD) is a common and costly reason for hospitalization and rehospitalization. The Hospital Readmissions Reduction Program penalizes hospitals for excess, nonelective, all-cause 30-day, inpatient rehospitalizations for COPD. We sought to determine how broadening the outcome definition would alter the numbers of patients being counted, specifically if observation stays and patients who died in the post-discharge period were included.

Methods: We performed a retrospective cohort study of patients hospitalized for COPD between July 1, 2010 and December 31, 2017 in 21 hospitals in the Kaiser Permanente Northern California health care system. We classified encounters into 3 outcomes groups based on a 30-day post-discharge observation period: Group (1) non-elective, all-cause, inpatient rehospitalizations, which is the current metric; Group (2) composite outcome of Group 1 or all-cause mortality; and Group (3)composite outcome of Group 1 or non-elective, allcause, observation rehospitalization. We used the Box-Cox method to find the transformation of the cumulative curves that resulted in the smallest mean standard error. We used the slope of the transformed curve against days to test for significant differences between pairs of cumulative density curves.

Results: Of 1,384,025 hospitalizations, 11,304 encounters from 8097 patients met criteria to be index hospitalizations. The event rate for non-elective, all-cause, inpatient rehospitalizations was $17.1 \%$ (95\% CI 10.4-26.5). The event rate for all-cause mortality was $4.7 \%$ (95\% CI 3.1-7.7). The event rate for non-elective observation rehospitalizations was 3.9\% (95\% CI 1.7-7.0). The slope and standard error for Group 1 were 1.17 and 0.01 , respectively, while the slope and standard error for Group 2 were 1.62 and 0.01 , respectively $(P=0.02$ comparing Groups 1 and 2). The slope and standard error for Group 3 were 1.45 and 0.01 , respectively $(P=0.02$ comparing Groups 1 and 3).

Conclusion: We show that adding outcomes such as mortality and observation rehospitalizations would change the counts of patients contributing to the Hospital Readmission Reduction Program penalty for COPD if the outcome were broadened. Including mortality or observation stays in the quality incentive program might incentivize hospitals and providers to prevent these events in addition to inpatient rehospitalizations.

\footnotetext{
Abbreviations: chronic obstructive pulmonary disease, COPD; Hospital Readmissions Reduction Program, HRRP; Centers for Medicare and Medicaid Services, CMS; Comorbidity Point Score, version 2, COPS2; Laboratory-based Acute Physiology Score, version 2, LAPS2; skilled nursing facility, SNF

Funding Support: This work was funded by The Permanente Medical Group (Oakland, California). Dr. Camargo was supported by grant R01 HS-023305 from the Agency for Healthcare Research and Quality (Rockville, Maryland).

Date of Acceptance: August 14, 2020 | Published Online Date: November 6, 2020

Citation: Myers LC, Camargo C, Escobar G, Liu VX. Expanding post-discharge readmission metrics in patients with chronic obstructive pulmonary disease. Chronic Obstr Pulm Dis. 2021;8(1):54-59. doi: https://doi.org/10.15326/jcopdf.2020.0160
} 
1 Division of Research, Kaiser Permanente Northern California, Oakland, California, United States

2 The Permanente Medical Group, Oakland, California, United States

3 Department of Emergency Medicine, Massachusetts General Hospital, Boston, Massachusetts, United States

\section{Address correspondence to:}

Laura Myers, MD, MPH

Division of Research

Kaiser Permanente Northern California

2000 Broadway

Oakland, CA 94612

Email: laura.c.myers@kp.org

Telephone: (925) 433-3491

\section{Keywords:}

chronic obstructive pulmonary disease; rehospitalizations; care quality

\section{Background}

Chronic obstructive pulmonary disease (COPD) causes 700,000 hospitalizations per year at an annual cost of $\$ 18$ billion. 1,2 Approximately $20 \%$ of these hospitalizations result in rehospitalizations within 30 days. ${ }^{3,4}$ Under the Hospital Readmissions Reduction Program (HRRP), ${ }^{5}$ hospitals experience financial penalties for excess rehospitalizations occurring after index hospitalizations for various target conditions, including COPD.

Many have criticized how the Center for Medicare and Medicaid Services (CMS) counts rehospitalizations under the HRRP. For example, CMS does not penalize hospitalizations with "observation status" under the HRRP. Additionally, CMS does not treat death as a competing risk. In other words, patients who die before being rehospitalized are not counted in the metric. It is unclear whether adding either outcome would impact the raw counts of COPD patients counted in the quality metric.

We sought to determine how broadening the HRRP outcome definition would alter the numbers of patients being counted if patients were included who (1)were rehospitalized under observation status (not admission status) and/or (2) died in the 30-day postdischarge window.

\section{Methods}

\section{Study Design and Data Source}

We performed a retrospective cohort study of patients hospitalized for COPD and examined the events occurring in the 30-day post-discharge window. The goal was to determine if including events, such as observation hospitalizations and death, might alter the number of patients counted in the HRRP quality metric for COPD. We included adults (age $\geq 18$ years) hospitalized between July 1, 2010 and December 31, 2017 in 21 hospitals in the Kaiser Permanente Northern California system, an integrated health care system serving 4.4 million members with Medicare Advantage, Medi-Cal and/or the Kaiser Foundation Health Plan. Kaiser Permanente's institutional review board approved this work (CN-17-2876_02). They provided a waiver of informed consent.

\section{Cohort Formation}

Index hospitalizations had COPD as the primary discharge diagnosis based on the Agency for Healthcare Research and Quality's Clinical Classification Software (Hypergroup 4, Subcategory 127). ${ }^{6}$ We excluded patients with bronchiectasis codes (J470/J471/J479/494/4940/4941). Index hospitalizations could be inpatient or observation hospitalizations according to our group's previous work. ${ }^{7}$ Given that observation stays and death are rare events, we allowed rehospitalizations to be index hospitalizations as well as outcome events, i.e., patients could contribute multiple index hospitalizations if the inclusion and exclusion criteria were met. This approach has been done previously and maximizes power. ${ }^{7}$

We required that patients survive index hospitalizations. We also required that patients either (1)be a health plan member for 30 days after discharge from index hospitalizations or (2)have terminal events (death) in the 30 -day post period. These criteria ensured that patients were eligible for the outcomes described below and were not lost to follow-up. We did not require continuous membership in the year prior to the hospitalization in order to maximize sample size, and because turnover within the Kaiser Permanente Northern California health care system is limited. ${ }^{8}$ Patients transported into Kaiser hospitals for rehospitalizations were excluded because decisions to rehospitalize were made by 
outside institutions; these were rare $(<6 \%)$.

\section{Outcome Groups}

We classified encounters into the following outcome groups based on data from the 30-day post-discharge follow-up period:

Group (1) non-elective, all-cause inpatient rehospitalizations, which is the current metric used in the HRRP ${ }^{3,5,9}$;

Group (2) composite outcome comprised of nonelective, all-cause inpatient rehospitalization or all-cause mortality;

Group (3) composite outcome comprised of nonelective, all-cause inpatient rehospitalization or non-elective all-cause observation rehospitalization.

We defined rehospitalizations as "nonelective" according to federal definitions put forth by CMS, which we have also used in previous work. ${ }^{7}$ Rehospitalizations that began as observation rehospitalizations and converted to inpatient rehospitalizations were considered inpatient rehospitalizations.

\section{Statistical Methods}

In order to find the best fitting curve of the cumulative distribution lines as a function of days, we used the Box-Cox method to find the transformation of the cumulative curves that resulted in the smallest mean standard error. ${ }^{10}$ This analysis showed that the best fitting curves were not linear when mortality or observation stays were included as an outcome. However, since the relationships between the transformed curve and days were linear, we used the slope of the transformed curve against days to test for significant differences between pairs of cumulative density curves for the various measures. We show the curves with 95\% confidence intervals. Because we had separate hypotheses for adding mortality and observation stays, we did not adjust for multiple comparisons.

We performed the analysis at the encounter-level in order to understand the impact of expanding the definition of encounter-level outcome events, not hospital-level rates. We examined counts because we wanted to understand if the raw counts would change. We stratified the results before and after 2015, which was when COPD became a target condition in the HRRP.
We used the transreg procedure in SAS (version 9.4, Cary, North Carolina). ${ }^{11}$

\section{Results}

Of 1,384,025 hospitalizations, 11,304 encounters from 8097 patients met criteria to be index hospitalizations. Most index hospitalizations were inpatient hospitalizations (74.8\%) versus observation hospitalizations (25.2\%). We report the baseline characteristics of the cohort (Table 1). The mean age of patients was 73.5 years (95\% CI 70.7-76.8). The cohort consisted of $19.8 \%$ current smokers and $66.9 \%$ previous smokers. The median Charlson score was 3 (interquartile range 3-4). The severity of illness at the time of hospitalization was Laboratory Acute Physiology Score, version $2^{12,13}$ of 80.3 (95\% CI 72.6-85.9). The mean length of stay was 4.1 days (95\% CI 3.5-5.2 days). Most patients had advanced care directives of full code at the time of discharge (68.6\%). Most patients were discharged home (74.6\%) with only $3.6 \%$ referred to hospice.

The individual and composite outcome rates are listed in Table 1. The event rate for non-elective, all-cause inpatient rehospitalizations (Group 1) was $17.1 \%$ (95\% CI 10.4-26.5). The event rate for all-cause mortality was $4.7 \%$ (95\% CI 3.1-7.7). The event rate for non-elective observation rehospitalizations was $3.9 \%$ (95\% CI 1.7-7.0). The outcome rate in Group 2 was $20.0 \%$ (95\% CI 11.9-28.3). The outcome rate in Group 3 was $20.0 \%$ (95\% CI 13.0-30.1).

We show the differences between groups over the 30-day post-discharge period. First, we show the difference in groups depending on whether mortality was included in the outcome. The slope and standard error for Group 1 were 1.17 and 0.01, respectively, while the slope and standard error for Group 2 were 1.62 and 0.01 , respectively $(P=0.02$ comparing Groups 1 and 2, Figure 1a). Next, we show the difference in groups depending on whether observation rehospitalizations were included in the outcome. The slope and standard error for Group 3 were 1.45 and 0.01 , respectively ( $P=0.02$ comparing Groups 1 and 3, Figure 1b). The sensitivity analysis showed that these findings were consistent before and after 2015 . 


\section{Table 1. Baseline Characteristics}

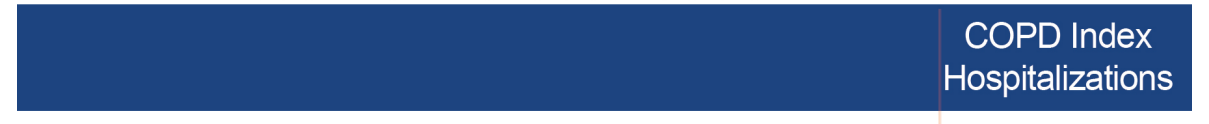

\begin{tabular}{|c|c|}
\hline Age, years & $73.5(70.7-76.8)$ \\
\hline Sex (\% male) & $43.8(35.5-59.1)$ \\
\hline \multicolumn{2}{|l|}{ Tobacco Smoking Status } \\
\hline Current & $19.8(13.2-24.9)$ \\
\hline Never & $10.2(5.0-14.6)$ \\
\hline Quit & $66.9(60.2-73.2)$ \\
\hline Unknown & $3.1(2.2-4.9)$ \\
\hline Admission via Emergency Department & $98.1(95.1-100)$ \\
\hline Charlson Score & $3.0(3.0-4.0)$ \\
\hline Charlson Score $\geq 4$ & $46.4(39.9-55.5)$ \\
\hline COPS2 $^{\mathrm{a}}$ & $76.3(68.1-90.2)$ \\
\hline COPS2 $\geq 65$ & $55.9(48.2-65.8)$ \\
\hline Admission LAPS2 $^{\text {b }}$ & $80.3(72.6-85.9)$ \\
\hline Discharge LAPS2 & $61.9(51.6-67.8)$ \\
\hline LAPS $2 \geq 110$ & $15.0(10.4-20.0)$ \\
\hline Full Code at Discharge & $68.6(55.7-78.0)$ \\
\hline Length of Stay, days & $4.1(3.5-5.2)$ \\
\hline \multicolumn{2}{|l|}{ Discharge Disposition } \\
\hline Regular Discharge & $74.6(55.3-85.6)$ \\
\hline Home Health & $19.6(9.5-38.2)$ \\
\hline Regular SNF & $4.6(1.7-9.6)$ \\
\hline Custodial SNF & $1.2(0.2-4.1)$ \\
\hline Hospice Referral at Time of Discharge & $3.6(1.4-8.0)$ \\
\hline \multicolumn{2}{|l|}{ Outcomes } \\
\hline \multicolumn{2}{|l|}{ Individual } \\
\hline Group 1: Non-elective inpatient rehospitalization & $17.1(10.4-26.5)$ \\
\hline 30-day post-discharge mortality & $4.7(3.1-7.2)$ \\
\hline Non-elective observation rehospitalization & $3.9(1.7-7.0)$ \\
\hline \multicolumn{2}{|l|}{ Composite } \\
\hline Group 2: Non-elective inpatient rehospitalization or death & $20.0(11.9-28.3)$ \\
\hline Group 3: Non-elective inpatient or observation rehospitalization & $20.0(13.0-30.1)$ \\
\hline
\end{tabular}

${ }^{a}$ The COPS2 score reflects comorbidity burden and is assigned based on all diagnoses incurred by a patient in the 12 months preceding the index hospitalization. The univariate relationship of COPS2 with 30-day mortality is as follows: $0-39,1.7 \% ; 40-64,5.2 \% ; 65+, 9.0 \%$.

${ }^{b}$ The LAPS2 score reflects severity of acute illness and is assigned based on a patient's worst vital signs, pulse oximetry, neurological status, and 16 laboratory test results in the 72 hours preceding hospitalization. The univariate relationship of an admission LAPS2 with 30-day mortality is as follows: $0-59,1.0 \%$; $60-109,5.0 \% ; 110+, 13.7 \%$.

COPS2=Comorbidity Point Score, version 2; LAPS2=Laboratory-based Acute Physiology Score, version 2; $\mathrm{SNF}=$ skilled nursing facility

\section{Discussion}

We show that adding outcomes such as mortality and observation rehospitalizations would change the counts of patients meeting criteria for the HRRP metric for COPD. We believe this is an important finding for multiple reasons. First, the rates of mortality and observation rehospitalizations were low $(<5 \%)$ yet including them would change the counts of patients flagged by the metric. Second, quality metrics used in programs such as the HRRP induce behavior change by incentivizing hospitals and providers. Knowing the effect of these incentives and whether changing the definitions of the outcomes might incentivize different behavior is critical for developing effective reimbursement policies. Including mortality or observation stays in the outcome of interest could incentivize hospitals and providers to focus on preventing these events too.

As previously mentioned, CMS does not penalize hospitalizations recorded as observation status under the HRRP. There is controversy as to whether observation hospitalizations have changed in response to the policy for any of the target conditions. Some evidence suggests that they did increase regardless of condition treated in the index stay, ${ }^{7}$ while other evidence using Medicare data shows the opposite. ${ }^{14}$ Additionally, CMS does not treat death as a competing risk. In other words, patients who die before being rehospitalized are not counted in the metric. There is controversy about whether this adversely incentivizes hospitals not to readmit patients, even if they are at high risk of death. Some evidence actually suggests an association between increased mortality and HRRP for patients with heart failure and pneumonia, ${ }^{15}$ but another study shows that death rates are low for heart failure and pneumonia and randomly distributed among hospital types. ${ }^{16}$ Neither outcome has been assessed in COPD, which prompted this study.

The study has limitations and strengths. First, we examined patients during an 8-year period, during which CMS implemented the policy, but findings were similar before and after COPD became a target 


\section{Figure1a. Addition of 30-day All-Cause Mortality to All-Cause Inpatient Rehospitalization Metric}

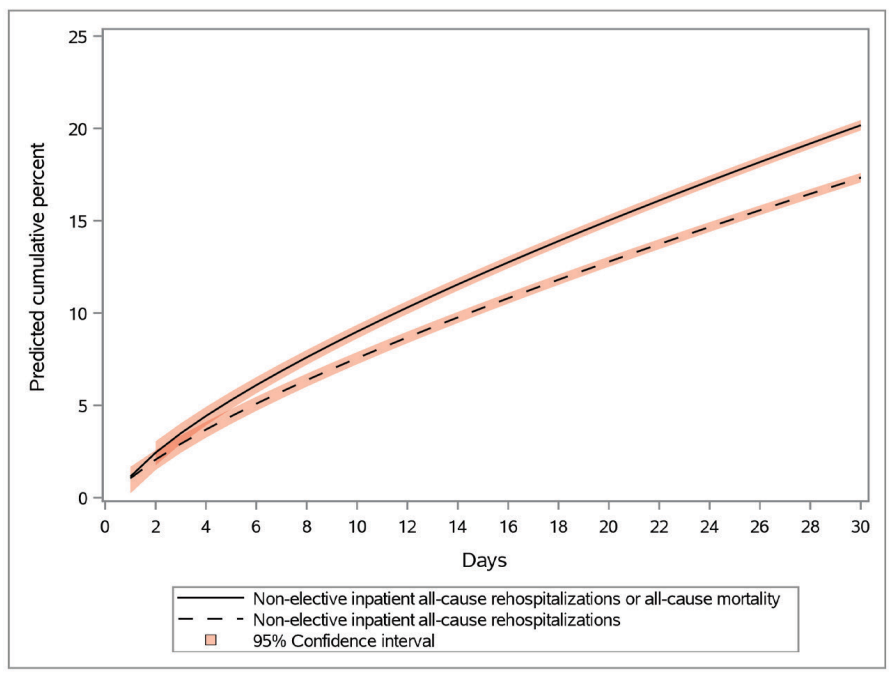

The lower curve represents the percentage of patients over time who have inpatient rehospitalizations for any reason (slope 1.17, standard error 0.01). The upper curve represents the percentage of patients over time who have either inpatient rehospitalizations for any reason or all-cause mortality (slope 1.62, standard error $0.01)$. $P$ value for this comparison of slopes is 0.02 .

\section{Figure 1b. Addition of 30-day All-Cause Observation Rehospitalizations to All-Cause Inpatient Rehospitalization \\ Metric}

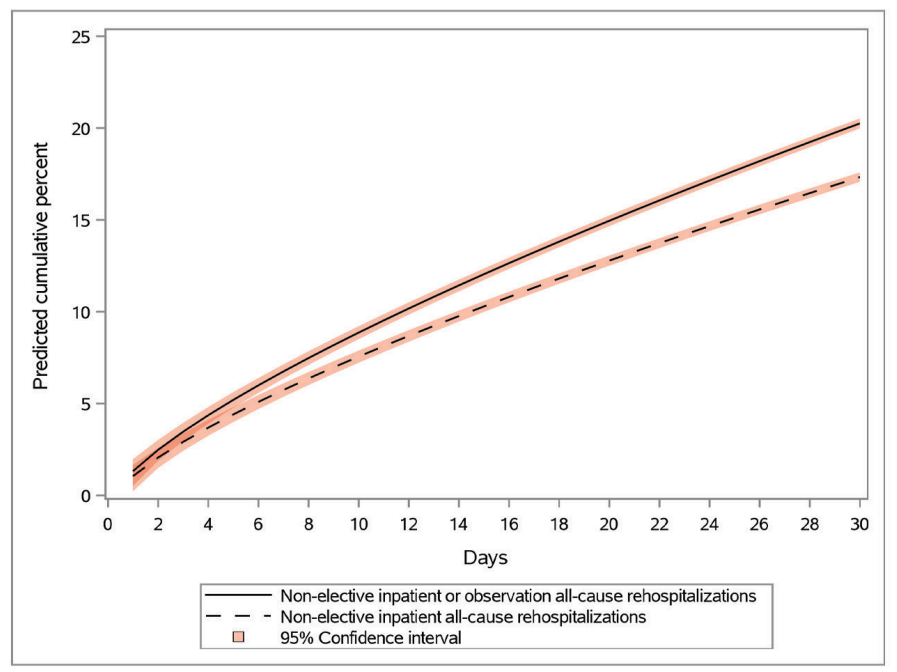

The lower curve represents the percentage of patients over time who have inpatient rehospitalizations for any reason (slope 1.17, standard error 0.01). The upper curve represents the percentage of patients over time who have either inpatient rehospitalizations for any reason or all-cause observation rehospitalizations (slope 1.45, standard error 0.01). $P$ value for this comparison of slopes is 0.02 . condition. Second, Kaiser Permanente Northern California serves a small number of fee-for-service Medicare patients but a large number of Medicare Advantage patients. We felt that these results were applicable to the Medicare population being counted in the current HRRP metric, given that mean age was approximately 74 years, and the allcause non-elective inpatient rehospitalization rate was consistent with other reports examining COPD readmissions (approximately 18\%-20\%). ${ }^{4,17}$ Using our data as opposed to administrative data allowed us to include granular, longitudinal data elements, like smoking status, referral to hospice and lab-based severity of illness score to describe the population being studied.

In summary, the counts of patients change significantly if the HRRP quality metric for COPD includes patients who died or were rehospitalized under observation status, despite the outcome rates for these 2 outcomes being low. Further research is needed to understand how hospital-level risk adjusted rates for Medicare patients would change if the metric were to be expanded and whether restricting the measurement to cause-specific rehospitalizations (respiratory-related, for example) would change the behavior of hospitals and providers to focus on more specific, respiratory-related prevention strategies.

\section{Acknowledgments}

The authors wish to acknowledge Colleen Plimier, MPH who did the data extraction and Patricia Kipnis, $\mathrm{PhD}$ who consulted on the statistical analysis.

Author contributions: LCM designed the study, conducted the analysis, drafted the manuscript and revised the manuscript in response to feedback. CC designed the study and interpreted the results. GE provided institutional review board approval, oversaw data extraction and interpreted the results. VXL designed the study, interpreted the results and critically revised the manuscript. All authors contributed to the intellectual content of the article and gave final approval of the version being published.

\section{Declaration of Interest}

The authors do not have any conflicts of interest or disclosures. 


\section{References}

1. Perera PN, Armstrong EP, Sherrill DL, Skrepnek GH. Acute exacerbations of COPD in the United States: inpatient burden and predictors of costs and mortality. COPD. 2012;9(2):131-141. doi: https://doi.org/10.3109/15412555.2011.650239

2. Ford ES. Hospital discharges, readmissions, and ED visits for COPD or bronchiectasis among US adults: findings from the Nationwide Inpatient Sample 2001-2012 and Nationwide Emergency Department Sample 2006-2011. Chest. 2015;147(4):989-998.

doi: https://doi.org/10.1378/chest.14-2146

3. Feemster LC, Au DH. Penalizing hospitals for chronic obstructive pulmonary disease readmissions. Am J Respir Crit Care Med. 2014;189(6):634-639.

doi: https://doi.org/10.1164/rccm.201308-1541PP

4. Jacobs DM, Noyes K, Zhao J, et al. Early hospital readmissions after an acute exacerbation of chronic obstructive pulmonary disease in the Nationwide Readmissions Database. Ann Am Thorac Soc. 2018;15(7):837-845.

doi: https://doi.org/10.1513/AnnalsATS.201712-913OC

5. Centers for Medicare and Medicaid Services (CMS). Hospital Readmissions Reduction Program. CMS website. Published 2019. Accessed May 14, 2019. https://www.cms.gov/medicare/medicarefee-for-service-payment/acuteinpatientpps/readmissions-reductionprogram.html

6. Agency for Healthcare Quality and Research Healthcare Cost and Utilization Project (HCUP). Clinical Classification Software. HCUP website. Published 2015. Updated March 2016. Accessed March 19, 2020. https://www.hcup-us.ahrq.gov/toolssoftware/ccs/CCSUsers Guide.pdf

7. Escobar GJ, Plimier C, Greene JD, Liu V, Kipnis P. Multiyear rehospitalization rates and hospital outcomes in an integrated health care system. JAMA Netw Open. 2019;2(12):e1916769.

doi: https://doi.org/10.1001/jamanetworkopen.2019.16769

8. Gordon N, Lin T. The Kaiser Permanente Northern California adult member health survey. Perm J. 2016;20(4):15-225.

doi: https://doi.org/10.7812/TPP/15-225

9. Grosso LM, Lindenauer P, Wang C, et al. Hospital-level 30-day readmission following admission for an acute exacerbation of chronic obstructive pulmonary disease. In: Measure Methodology Report. 2011.

10. Draper N, Smith H. Applied Regression Analysis. 3rd ed. John Wiley \& Sons; 1998. doi: https://doi.org/10.1002/9781118625590

11. SAS/STAT User's Guide. The TRANSREG Procedure. Example 119.2 Box-Cox SAS Institute website. Accessed July 2, 2020. https:// documentation.sas.com/?docsetId=statug\&docsetTarget=statug transreg_details02.htm\&docsetVersion=14.2\&locale=en
12. Escobar GJ, Greene JD, Scheirer P, Gardner MN, Draper D, Kipnis P. Risk-adjusting hospital inpatient mortality using automated inpatient, outpatient, and laboratory databases. Med Care. 2008;46(3):232-239. doi: https://doi.org/10.1097/MLR.0b013e3181589bb6

13. Escobar GJ, LaGuardia JC, Turk BJ, Ragins A, Kipnis P, Draper D. Early detection of impending physiologic deterioration among patients who are not in intensive care: development of predictive models using data from an automated electronic medical record. $J$ Hosp Med. 2012;7(5):388-395. doi: https://doi.org/10.1002/jhm.1929

14. Zuckerman RB, Sheingold SH, Orav EJ, Ruhter J, Epstein AM. Readmissions, observation, and the Hospital Readmissions Reduction Program. NEngl J Med. 2016;374(16):1543-1551. doi: https://doi.org/10.1056/NEJMsa1513024

15. Wadhera RK, Joynt Maddox KE, Wasfy JH, Haneuse S, Shen C, Yeh RW. Association of the Hospital Readmissions Reduction Program with mortality among Medicare beneficiaries hospitalized for heart failure, acute myocardial infarction, and pneumonia. JAMA. 2018;320(24):2542-2552. doi: https://doi.org/10.1001/jama.2018.19232

16. Papanicolas I, Orav EJ, Jha AK. Is mortality readmissions bias a concern for readmission rates under the Hospital Readmissions Reduction Program? Health Serv Res. 2020;55(2):249-258. doi: https://doi.org/10.1111/1475-6773.13268

17. Myers LC, Faridi MK, Hasegawa K, Hanania NA, Camargo CA, Jr. The Hospital Readmissions Reduction Program and readmissions for chronic obstructive pulmonary disease, 2006-2015. Ann Am Thorac Soc. 2019;17(4). doi: https://doi.org/10.1513/AnnalsATS.201909-672OC 